\title{
THE EFFECTIVENESS OF SPINAL MANIPULATIVE THERAPY (SMT) IN THE MANAGEMENT OF NON SPECIFIC LOW BACK PAIN (LBP)
}

\section{Dr. Scalella Roberto}

Dr. Physiotherapist Cert. SMT (Spinal Manipulative Therapy) Freelance Physiotherapist at "Studio di Fisioterapia CAMIGRA" in Naples (Italy)

KEYWORDS: Spinal Manipulation, Effects of High Velocity Low Amplitude Thrust Manipulation Techniques (HVLA), Low back pain randomized clinical trial, Chronic Low back pain, Acute Low back pain, Lumbar Spine Manual Therapy, Low back pain treatment outcome, Manipulation Randomized controlled trials, Comparative Study, Review literature, Meta-Analysis,

\section{ABSTRACT}

A systematic revie $w$ of a number of studies in order to verify the real effectiveness of Spinal Manipulative Therapy (SMT) for management of nonspecific Low Back pain in order to relieve pain, improve function and diminish disability.

It is known that the diagnosis of low back pain is a difficult matter, due to different causes related to it, not always readily apparent, considering the main distinction between specific and non-specific low back pain, in this review, a special attention has been dedicated on the second one.

Despite many published randomized clinical trials, a substantial number of reviews and several clinical guidelines, the real effectiveness of this therapy to manage nonspecific low back pain is still minimal.

Following the most recent North American Spine Society (NASS) guidelines, this review considers selected and high quality studies with more stringent criteria of evidence-based admissibility, and by the way, a special attention has been paid on the studies that, more than other, tried to assess the effect of spinal manipulation SMT compared to other kind of therapies in patients with nonspecific LBP. It should be noted that this study is designed to be a simple review of the best evidence based recommendations so it should not be seen as a standard of care, or a treatment protocol with type, frequency and duration of intervention available for everyone.

\section{PURPOSE}

To assess the benefits and harms of spinal manipulative therapy (SMT) in order to relieve pain, improve function and diminish disability in patients with non-specific low back pain and though, to provide a "tool" to assist Physiotherapists in their clinical decision-making processes.

\section{DATA SOURCES}

Medline, PubMed, Embase, Cochrane Central Register of Controlled Trials (CENTRAL), CINAHL, Physiotherapy Evidence Database (PEDro), Index to Chiropractic Literature, and trial registries up to 2020.

\section{METHOD}

A Systematic Review and a bibliographic research of the most high-quality Randomized and Controlled Clinical Trials. Focus has been mainly directed on the studies of the North American Spine Society (NASS) with more stringent criteria of Evidence-based admissibility in order to isolate the effect of spinal manipulative therapy SMT.

\section{BACKGROUND}

Low back pain is defined as pain of musculoskeletal origin extending from the lowest rib to the gluteal fold that may at times extend as somatic referred pain into the thigh (above the knee).
Nowadays Low-back pain is an increasing problem in health care. LBP is one of the most common and often disabling disorder. It is usually cause of medical consultations that frequently turn into drug therapies.

At least 80 percent of people worldwide will experience low back pain once in their lifetime. A recent systematic review of the clinical course of non-specific low back pain found that in the first 3 months, 33\% of patients showed recovery, but 1 year after onset, $65 \%$ still reported pain.

It Is probably the most common cause of job-related disability and worsening of life quality of the single person which turns into a great increase in healthcare system and society costs.

Despite the high frequency of low back pain, finding real causes and effective therapeutic programs remain highly problematic. Part of this problem is due to the nature of low back pain. There are many different causes of low back pain and not always are readily apparent. In fact, with the exception of sciatica (resulting from entrapment of the spinal nerve root by degenerative changes or by disc herniation) most causes of low back pain lack objective clinical signs.

These obscure causes are responsible for most of the back pain seen in clinical practice. It is important to remember that less than $10 \%$ of low back pain is due to herniation of the intervertebral disc or entrapment of spinal nerves by degenerative disc disease. 
Accordingly, the diagnosis of low back pain is a difficult matter, and since most low back pain is nonspecific, the real effects of many commonly applied therapies remain hard to demonstrate.

Nowadays statistics say that one fourth to one half of all patients treated in physical therapy clinics suffer from nonspecific low-back pain.

The most frequent causes are strains on muscles and ligaments in the back due to excess activity with a production of pain and stiffness in the lower back, as well as muscle spasms, disc injury (we know that discs in the back are usually prone to injury, and this risk increases with age), sciatica, as a result of herniated disc that presses on the sciatic nerve producing pain in the legs and feet usually like burning and other symptoms such as numbness, cramping or weakness, spinal stenosis (when the spinal column narrows, putting pressure on the spinal cord and spinal nerves), abnormal spine curvatures, usually diagnosed during childhood or adolescence such as Scoliosis, kyphosis or lordosis that causes pain and poor posture by placing pressure on muscles, tendons, ligaments, vertebrae.

Less frequently we have a specific low back pain caused by a number of other conditions such as tumors, inflammatory conditions such as spondyloarthritis, arthritis or fibromyalgia.

In these kind of LBP is crucial to shortly identify clinical clues, or "red flags" and prompt further evaluation or imaging. Red flags include cancer, unexplained weight loss, immunosuppression, intravenous drug use, urinary tract infection, fever, and significant trauma relative to age bladder or bowel incontinence urinary retention with overflow incontinence.

In most cases of low back pain, diagnostic testing such as X-ray studies, bone scan, computed tomography (CT) scan and magnetic resonance imaging (MRI) are not required. X-ray imaging or other diagnostic tests may be advised in cases of pain associated with severe trauma, history of cancer, fever or other medical problems.

Imaging on initial presentation should be reserved when there is suspicion for cauda equina syndrome, malignancy, fracture, or infection. Plain radiography of the lumbar spine is appropriate to assess fracture and bony abnormality, whereas magnetic resonance imaging is better for identifying the source of neurologic or soft tissue abnormalities.

Anyway it is important to realize that the diagnosis is based on a combination of history, physical examination and diagnostic testing, not imaging tests alone.

Low back pain is defined acute when pain is present for few days up to six weeks. It may be experienced as aching, burning, stabbing, sharp or dull, well-defined, or vague. The intensity may range from mild to severe and may fluctuate. The pain may radiate into one or both buttocks or even into the thigh/hip area. No matter which tissue is initially irritated, a cascade of events occurs which contributes to the pain experience. Usually the acute pain subsides spontaneously over time and the originally irritated tissue heals. Fifty percent of episodes nearly completely resolve within two weeks, and $80 \%$ by six weeks.

Instead Low back pain is considered to be chronic if it has been present for greater than three months. The type of pain may vary greatly and may be felt as bone pain, nerve pain or muscle pain. The sensation of pain may also vary, and the intensity may range from mild to severe. Chronic back pain ultimately is more disabling and dispiriting because of the lasting physical impediment that causes moreover psychological effects.

\section{- STUDY SELECTION CRITERIA}

A number of studies has been reviewed several times following the NASS indications and, by the way, it is essential to remember how NASS selected studies and practitioners. First of all, developers complete a preliminary program of Evidence-Based Medicine Training including a series of readings and exercises in order to be ready on systematically evaluating literature and developing evidence-based guidelines. Standardized levels of evidence and grades of recommendation has been adopted to assist practitioners in easily understanding the real strength of the evidence and recommendations within the guidelines.

The levels of evidence range from Level I (high quality randomized controlled trial) to Level V (expert consensus). Grades of recommendation indicate the strength of the recommendations made in the guideline based on the quality of the literature. (insert Table 1 Here)

Grades of Recommendation for or against intervention are the following:

A: Good evidence (Level I studies with consistent findings)

B: Fair evidence (Level II or III studies with consistent findings)

C: Poor quality evidence (Level IV or V studies)

I: Insufficient or conflicting evidence not allowing a recommendation

Basically, "A" recommendations indicate a test or intervention is "recommended";

"B" recommendations "suggest" a test or intervention and "C" recommendations indicate a test or intervention "may be considered" or "is an option"; "I" or "Insufficient Evidence" statements clearly indicate that "there is insufficient evidence to make a recommendation for or against" a test or intervention. (insert Table 2 here)

NASS guideline development process also Indicated Inclusion/Exclusion criteria, and followed 15 different work steps.

Inclusion criteria are: Adult patients aged 18 and older, patients with low back pain limited to somatic referred pain/non-radicular pain limited to above.

(people with Leg pain were excluded because it might depend from specific causes, including disc herniation that need a separate evaluation of the evidence).

Exclusion criteria are: patients less than 18 years of age, and/or with Red Flags such as Tumor, Infection, Metabolic disease, Inflammatory arthritis, Fracture or Patients with a diagnosed deformity, including spondylolisthesis, spondylolysis and scoliosis, Patients with Extra-spinal conditions such as visceral, vascular, genito-urinary, presence of neurological deficit, pregnancy. The work steps have been the following: Step 1) Recruitment of Guideline Members and Involvement of Stakeholder Representatives Step 2) Identification of Work Groups. Step 3) Surveying Patients. Step 4) Identification of Clinical Questions. Step 5) External Review of Clinical Question Protocol. Step 6) Identification of Search Terms and Parameters. Step 7) Completion of the Literature Search. Step 8) Review of Search Results/Identification of Literature to Review. Step 9) Evidence Analysis. Step 10) Formulation of Evidence-Based Recommendations and Incorporation 
of Expert Consensus. Step 11) Internal Review of Draft Guideline. Step 12) External Review of Draft Guideline. Step 13) Submission for Board Approval. Step 14) Submission for Publication. Step 15) Review and Revision Process.

The result has been a very reliable "guideline panel" consisting on seven sections: Diagnosis, Imaging, Medical and Psychological Treatment, Interventional Treatment, as a Physical Medicine and Rehabilitation, Surgical Treatment and Cost-Utility.

As Physiotherapist Cert. SMT, my focus has been on the "Physical Medicine and Rehabilitation" panel but, I suggest to all physiotherapists who practice SMT to read the entire NASS document.

\section{STUDIES SELECTED}

A special attention has been paid on the studies that, more than other, tried to assess the effect of spinal manipulation SMT compared to other kind of therapies .

By the way, from the latest document produced by NASS, 6 randomized controlled trials (with Level I or II therapeutic evidence) have been identified with results in which we could put some confidence. In these studies, treatments were delivered by a variety of practitioners, including chiropractors, manual therapists and osteopaths.

The most important trial reviewed are the Following:

1. Haas, conducted a randomized controlled trial in patients with chronic LBP to identify the doseresponse relationship between visits to a chiropractor for SMT and treatment outcomes and to compare treatment outcomes of manipulation versus light massage. Four hundred patients with nonspecific LBP for at least 3 months were prescribed 18 sessions with a chiropractor (three times a week for six weeks).

a. Participants were randomized to receive either $0,6,12$ or 18 sessions of SMT ( $n=100$ in each group). The chiropractor provided light massage for the remaining sessions that did not include SMT. Pain and disability (modified Von Korff pain and disability scales) were recorded at baseline and throughout a 52-week follow-up period. Pain and disability improved in all groups and was sustained for 52 weeks. The greatest

b. treatment effects were found from 12 sessions of SMT. The authors concluded that 12 SMT visits had modest effects on chronic LBP outcomes compared to the other dose levels, but the difference was not well distinguished.

c. This study offers Level I therapeutic evidence that, in patients with LBP, spinal manipulative therapy is associated with statistically significant improvement in pain compared to light massage. However, it is uncertain whether this was clinically significant. Regarding frequency, this study offers Level I therapeutic evidence that $12-18$ visits of SMT were statistically better than 6 visits at 12-week follow-up; no difference between doses at one-year follow-up. Again, the differences were small and the clinical significance was uncertain.

2. Hancock, In a placebo controlled randomized trial, evaluated the effectiveness of non-steroidal anti-inflammatory drugs (NSAIDS), spinal manipulative therapy (SMT), or combination of NSAIDs and SMT or the treatment of acute LBP. Patients presenting to their general practitioner with LBP were randomized into one of 4 groups: diclofenac $50 \mathrm{mg}$ twice daily and placebo SMT $(n=60)$; SMT and placebo drug $(n=60)$; diclofenac $50 \mathrm{mg}$ twice daily and SMT $(\mathrm{n}=60)$; or double placebo $(n=60)$ until recovery or up to 4 weeks. The primary outcome of "days to recovery" was recorded as the first pain-free day and the first of 7 consecutive days with a pain score of 0 or 1 . There were no significant differences in days to recovery between groups. The authors concluded that the addition of diclofenac or spinal manipulative therapy do not improve recovery in patients with acute LBP already receiving first-line care. This study offers Level I therapeutic evidence that, in patients with acute LBP, there was no difference in resolution of pain between SMT, NSAIDS and placebo.

3. Von Heymann, In a placebo and sham randomized controlled trial, evaluated the effectiveness of spinal high-velocity low-amplitude manipulation compared to treatment with a nonsteroidal anti-inflammatory drug. Patients with LBP for less than 48 hours were randomized to receive SMT and placebo drug $(n=37)$, sham SMT and $50 \mathrm{mg}$ tablets diclofenac three times a day $(\mathrm{n}=38)$, or sham SMT and placebo drug $(\mathrm{n}=25)$. Physical disability (Roland Morris Disability Questionnaire), function (SF-12), pain (VAS) off work time and rescue medication usage were recorded at baseline, 7-9 days after randomization and 12- week follow-up. Both intervention groups had better outcomes than the placebo group. The manipulation group experienced faster and more distinct reduction in pain and disability compared to the diclofenac group. There were no adverse effects, harm or unexpected untoward events in either group. The authors concluded that SMT was significantly better than diclofenac and placebo in the treatment of LBP. This study offers Level I therapeutic evidence that, in patients with acute LBP, SMT resulted in statistically significant short-term (9 days) improvement in pain compared to medication. The clinical significance was uncertain. The two groups were similar at medium term follow up.

4. Doran and Newell investigated the effectiveness of four different treatment options in patients with acute LBP. Participants were randomly assigned to receive at least 2 sessions of manipulation per week $(n=98)$, at least 2 sessions of physiotherapy per week $(n=104)$, corset applied on the first day of the trial $(n=93)$ or two paracetamol tablets every 4 hours $(n=100)$. Patients completed questionnaires to report whether their pain was worse, unchanged, improved, or completely relieved at the end of the 3-week trial, 3 weeks after the conclusion of the trial, 3 months after enrollment and one year after enrollment. The authors concluded that there were no significant differences between any of the four studied interventions. In critique, no validated outcomes were included in this study and outcome data is limited. Due to these reasons, the work group downgraded this study. This potential Level I study provides Level II therapeutic evidence that, in patients with acute LBP, SMT, physiotherapy, medication and corset resulted in similar improvements up to 3 months. 
Tab. 1

\begin{tabular}{|c|c|}
\hline \multicolumn{2}{|r|}{ THERAPEUTIC STUDIES LEVEL INVESTIGATING THE RESULTS OF TREATMENT } \\
\hline Level I & $\begin{array}{l}\text { - High quality randomized trial with statistically significant difference or no } \\
\text { statistically significant difference but narrow confidence intervals } \\
\text { - Systematic review of Level I RCTs (and study results were homogenous) }\end{array}$ \\
\hline Level II & $\begin{array}{l}\text { - Lesser quality RCT (eg, }<80 \% \text { follow-up, no blinding, or improper randomization) } \\
\text { - Prospective4 comparative study } \\
\text { - Systematic review2 of Level II studies or Level I studies with inconsistent results }\end{array}$ \\
\hline Level III & $\begin{array}{l}\text { - Case-control study } \\
\text { - Retrospective comparative study } 5 \\
\text { - Systematic review2 of Level III studies }\end{array}$ \\
\hline Level IV & Case series \\
\hline Level V & Expert opinion \\
\hline
\end{tabular}

Tab.2

\begin{tabular}{|c|l|l|l|}
\hline \multicolumn{2}{|l|}{ GRADE OF RECOMMENDATION } \\
\hline A & Good evidence & Two or more consistent Level I studies & Intervention is recommended \\
\hline B & Fair evidence & $\begin{array}{l}\text { One Level I study with additional } \\
\text { supporting Level II or III studies }\end{array}$ & Intervention is suggested \\
\hline C & Poor quality evidence & $\begin{array}{l}\text { One Level I, II, III or IV study } \\
\text { with supporting Level IV studies }\end{array}$ & $\begin{array}{l}\text { Intervention may be considered or is } \\
\text { an option }\end{array}$ \\
\hline I & $\begin{array}{l}\text { Insufficient or conflicting } \\
\text { evidence }\end{array}$ & $\begin{array}{l}\text { A single level I, II, III or IV study } \\
\text { without other supporting evidence }\end{array}$ & $\begin{array}{l}\text { Insufficient evidence to } \\
\text { make a recommendation }\end{array}$ \\
\hline
\end{tabular}

1. Cecchi, compared the effect of spinal manipulation, back school and individual physiotherapy on pain and disability outcomes in a randomized trial of patients with chronic LBP. Patients were randomized to receive 15 one-hour sessions of back school (group exercise, education/ergonomics) over the course of 3 weeks $(n=70), 15$ one-hour sessions of individual physiotherapy (exercise, passive mobilization and soft-tissue treatment) over the course of 3 weeks ( $n=70)$, or 4-6 20-minute sessions of spinal manipulation per week for 4-6 weeks $(n=70)$. Participants recorded pain (Pain Rating Scale) and disability (Roland Morris Disability Questionnaire) at baseline, discharge and follow-up at 3,6 and 12 months. All patients experienced improvement in pain and disability at discharge and 12-month follow-up. Function, pain recurrences and drug intake were significantly reduced compared to back school or physiotherapy. The authors concluded that spinal manipulation resulted in better improvements in pain and function compared to back school or individual physiotherapy. This study provides Level I therapeutic evidence that, in patients with LBP, SMT results in statistically significant improvement in pain and functional compared to multimodal physical therapy (baseline scores indicate mild pain). However, it is uncertain if these improvements are of clinical significance. It offers Level IV evidence that SMT results in improved pain and function.

2. Dougherty, in a randomized control trial, evaluated the effectiveness of spinal manipulative therapy (SMT) in older veterans with chronic LBP compared to a sham intervention. Veterans at least 65 years of age with LBP for at least 3 months were randomly allocated to groups to receive SMT $(n=69)$ or sham intervention $(n=67)$ twice weekly for 4 weeks. Pain (VAS, SF-36 pain subscale), disability (Oswestry Disability Index) and physical function (SF-36 subscale, Timed Up and Go) were recorded at baseline and after 5 and 12 weeks. Both groups experienced improvements in pain and disability at 5 and 12 weeks. Disability improved significantly more in the SMT group compared to the control group. The authors concluded that SMT resulted in greater improvement in disability but not pain at 12 weeks. This study offers Level I therapeutic evidence that, in patients with chronic LBP, there were similar improvements in pain when comparing SMT to sham. There was statistically significant improvement in function in the SMT group. However, it is uncertain as to whether these functional improvements were clinically significant.

\section{CONCLUSIONS}

Although, Spinal manipulative therapy is widely practiced to treat patients with LBP, strong evidence of benefit is often lacking.

At first, this review seems to demonstrate that SMT appears to be as effective as other common therapies prescribed for nonspecific low-back pain, such as, exercise therapy, massage, standard medical care or standard physiotherapy, but a better data synthesis suggests there is moderate evidence that SMT may produce a fair reduction in pain intensity and moderate increase in function so, "recommendations" regarding the use of SMT can be made with some confidence as a viable option for the treatment of low back pain.

Despite trial heterogeneity resulted very large, all studies shown there is a moderate evidence that SMT in acute low back pain provides more short-term pain relief than commonly used physical therapy treatment strategies. In these cases, spinal manipulative therapy was associated with modest, although statistically significant improvements in pain and function up to 6 weeks.

Anyway, one of the best advice for acute low back pain 
is to remain active as tolerated. The natural inclination may be to stay in bed avoid activity, but activity keeps blood and nutrients flowing to the affected area, inhibiting inflammation and reducing muscular tension. Instead, local application of heat or ice can only temporarily reduce pain.

In patients with chronic low back pain, there is limited to moderate evidence that SMT combined with back exercises is more effective than exercise ore classic physical therapy alone. In these cases multimodal programs may be promising options. It means that is important to include a patient education program. The patient should learn a variety of stretching and strengthening exercises to continue on his own at home. Usually, a home exercise program combined with SMT gives permanent and long lasting effects on pain and disability and increase stability and strength around the structures stressed.

In both acute and chronic low back pain, there is a limited evidence that SMT resulted superior to sham (placebo) treatment.

Furthermore, no serious complications and harm have been observed with SMT in all studies.

\section{REFERENCES}

1. North American Spine Society (evidence-based clinical guidelines for multidisciplinary spine care)

2. "diagnosis and treatment of low back pain". 2020 978-1-929988-65-5

3. Bronfort G, Maiers MJ, Evans RL, et al. Supervised exercise, spinal manipulation, and home exercise for chronic low back pain: a randomized clinical trial. Spine J. 2011;11(7):585-598.

4. Childs JD, Fritz JM, Flynn TW, et al. A clinical prediction rule to identify patients with low back pain most likely to benefit from spinal manipulation: A validation study. Ann Intern Med. 2004;141(12):920-928+I-939.

5. Peterson CD, Haas M, Gregory WT. A pilot randomized controlled trial comparing the efficacy of exercise, spinal manipulation, and neuro emotional technique for the treatment of pregnancy-related low back pain. Chiropractic \& manual therapies. 2012;20.

6. Siepe CJ, Heider F, Haas E, et al. Influence of lumbar intervertebral disc degeneration on the outcome of total lumbar disc replacement: A prospective clinical, histological, X-ray and MRI investigation. European Spine Journal. 2012;21(11):2287-2299.

7. Haas M, Groupp E, Muench J, et al. Chronic disease self-management program for low back pain in the elderly. Journal of Manipulative and Physiological Therapeutics. 2005;28(4):228-237.

8. Kladny B, Fischer FC, Haas e I. [Evaluation of specific stabilizing exercise in the treatment of low back pain and lumbar disk disease in outpatient rehabilitation]. Zeitschrift für Orthopädie und ihre Grenzgebiete. 2003;141(4):401-405.

9. Williams CM, Hancock MJ, Maher CG, McAuley JH, Lin CWC, Latimer J. Predicting rapid recovery from acute low back pain based on the intensity, duration and history of pain: A validation study. Eur J Pain. 2014;18(8):1182-1189 1188p.

10. Williams CM, Maher CG, Hancock MJ, et al. Low back pain and best practice care: A survey of general practice physicians. Arch Intern Med. 2010;170(3):271-277.

11. Cooper K, Smith BH, Hancock E. Patients' perceptions of self-management of chronic low back pain: evidence for enhancing patient education and support. Physiotherapy. 2009;95(1):43-50 48p.

12. Garcia AN, Costa Lda C, Hancock MJ, de Almeida MO, de Souza FS, Costa LO. Efficacy of the McKenzie method in patients with chronic nonspecific low back pain: a protocol of randomized placebo-controlled trial. Physical Therapy. 2015;95(2):267-273.

13. Halliday MH, Ferreira PH, Hancock MJ, Clare HA. A randomized controlled trial comparing McKenzie therapy and motor control exercises on the recruitment of trunk muscles in people with chronic low back pain: A trial protocol. Physiotherapy (United Kingdom). $2015 ; 101(2): 232-238$.

14. Hancock M. Approach to low back pain - physiotherapy. Australian family physician. 2014;43(3):117-118.

15. Hancock MJ, Maher CG, Latimer J, Herbert RD, McAuley JH. Independent evaluation of a clinical prediction rule for spinal manipulative therapy: a randomised controlled trial.

16. European spine journal : official publication of the European Spine Society, the European Spinal Deformity Society, and the European Section of the Cervical Spine Research Society. 2008;17(7):936-943.

17. Hancock MJ, Maher CG, Latimer J, et al. Assessment of diclofenac or spinal manipulative therapy, or both, in addition to recommended first-line treatment for acute low back pain: a randomised controlled trial. Lancet (London, England). 2007;370(9599):1638-1643.

18. Hancock MJ, Maher CG, Latimer J, et al. Manipulative therapy and/or NSAIDs for acute low back pain: Design of a randomized controlled trial [ACTRN012605000036617]. BMC Musculoskelet Disord. 2005;6 (no pagination)(57).

19. Macedo LG, Maher CG, Hancock MJ, et al. Predicting response to motor control exercises and graded activity for patients with low back pain: preplanned secondary analysis of a randomized controlled trial. Phys Ther. 2014;94(11):1543-1554.

20. Von Heymann WJ, Schloemer P, Timm J, Muehlbauer B. Spinal high-velocity low amplitude manipulation in acute nonspecific low back pain: a double-blinded randomized controlled trial in comparison with diclofenac and placebo. Spine (03622436). 2013;38(7):540548549 p.

21. Cecchi F, Molino-Lova R, Chiti M, et al. Spinal manipulation compared with back school and with individually delivered physiotherapy for the treatment of chronic low back pain: a randomized trial with one-year follow-up. Clinical Rehabilitation. 2010;24(1):26-36 $11 \mathrm{p}$.

22. Cecchi F, Negrini S, Pasquini G, et al. Predictors of functional outcome in patients with chronic low back pain undergoing back school, individual physiotherapy or spinal manipulation. European Journal of Physical \& Rehabilitation Medicine. 2012;48(3):371-378 378p.

23. Cecchi F, Molino-Lova R, Chiti M, et al. Spinal manipulation compared with back school and with individually delivered physiotherapy for the treatment of chronic low back pain: a randomized trial with one-year follow-up. Clinical Rehabilitation. 2010;24(1):26-36 $11 \mathrm{p}$.

24. Dougherty PE, Karuza J, Dunn AS, Savino D, Katz P. Spinal Manipulative Therapy for Chronic Lower Back Pain in Older Veterans: A Prospective, Randomized, Placebo-Controlled Trial. Geriatric Orthopaedic Surgery and Rehabilitation. 2014;5(4):154-164.

25. Dougherty PE, Karuza J, Savino D, Katz P. Evaluation of a modified clinical prediction rule for use with spinal manipulative therapy in patients with chronic low back pain: A randomized clinical trial. Chiropractic and Manual Therapies. 2014;22 (1) (no pagination)(22).

26. Bronfort G, Maiers MJ, Evans RL, et al. Supervised exercise, spinal manipulation, and home exercise for chronic low back pain: a randomized clinical trial. Spine J. 2011;11(7):585-598.

27. Cecchi F, Negrini S, Pasquini G, et al. Predictors of functional outcome in patients with chronic low back pain undergoing back school, individual physiotherapy or spinal manipulation. Eur J36 Phys Rehabil Med. 2012;48(3):371-378.

28. Chen J, Phillips A, Ramsey M, Schenk R. A case study examining the effectiveness of mechanical diagnosis and therapy in a patient who met the clinical prediction rule for spinal manipulation. Journal of Manual \& Manipulative Therapy (Journal of Manual \& Manipulative Therapy). 2009;17(4):216-220 215p.

29. Childs JD, Fritz JM, Flynn TW, et al. A clinical prediction rule to identify patients with low back pain most likely to benefit from spinal manipulation: A validation study. Ann Intern Med. 2004;141(12):920-928+I-939.

30. Clark BC, Goss Jr DA, Walkowski S, Hoffman RL, Ross A, Thomas JS. Neurophysiologic effects of spinal manipulation in patients with chronic low back pain. BMC Musculoskeletal Disorders. 2011;12(1):170-170 171p 